\title{
Correlation between resistance mechanisms in Staphylococcus aureus and cell wall and septum thickening
}

This article was published in the following Dove Press journal:

Infection and Drug Resistance

17 October 2017

Number of times this article has been viewed

\author{
Ana Belén García' \\ José Manuel Viñuela-Prieto' \\ Laura López-González' \\ Francisco Javier Candel ${ }^{1,2}$ \\ 'Department of Clinical Microbiology, \\ Hospital Clínico San Carlos, \\ Complutense University, Madrid, \\ Spain; ${ }^{2}$ Health Research Institute, \\ Hospital Universitario San Carlos, \\ Madrid, Spain
}

Purpose: The aim of the present study is to examine cell wall and septum thickening of methicillin-sensitive Staphylococcus aureus (MSSA), methicillin-resistant S. aureus (MRSA), and methicillin- and linezolid-resistant $S$. aureus (MLRSA) isolates by transmission electron microscopy to correlate the association of resistance mechanisms with major changes in the morphology of membrane or septum.

Materials and methods: MSSA, MRSA, and MLRSA strains obtained from clinical samples of an outbreak that occurred in 2010 at the Intensive Care Unit of our Hospital were thawed and sown at $37^{\circ} \mathrm{C}$ in blood agar overnight. After that, they were washed, pelleted, and treated with a fixer solution. Pellets were dehydrated and finally embedded in resin. Transmission electron microscopy was used to characterize cell wall and septum thickening in all isolates. The comparison between the measurements obtained for each group was performed by a Kruskal-Wallis test and a post hoc Dunn-Bonferroni's pairwise comparison method.

Results: Differences in cell wall and septum thickness were statistically significant $(P<0.001$ and $P<0.001$, respectively) between the three groups. Moreover, significant differences were detected in wall and septum thickness between the MSSA and MRSA strains $(P<0.001$ and $P<0.001$, respectively) and between the MSSA and MLRSA strains $(P<0.001$ and $P<0.001$, respectively) but not between the MRSA and MLRSA strains ( $P=0.386$ and $P=0.117)$.

Conclusion: In this analysis, we correlate the resistance mediated by alterations in the cell membrane of $S$. aureus (methicillin-resistant, for example) with a greater thickness of the wall or septum. The resistance added to linezolid did not determine significant changes in the characteristics of the wall or septum with respect to those strains resistant only to methicillin. Keywords: linezolid-resistant $S$. aureus, vancomycin intermediate $S$. aureus, VISA, protein synthesis inhibitor antibiotics, TEM

\section{Introduction}

Nowadays, multiple antibiotic resistance in Staphylococcus aureus is a very concerning problem of skin infection antibiotic management. The study of morphological characteristics of staphylococcal cells is one essential step to better understand and overcome resistance.

Mutations that led to changes in biosynthesis and metabolism of $S$. aureus cell wall are being described as an important mechanism of resistance to several antibiotic groups. An excess of wall material and decreased rates of autolysis are observed not only in isolates with resistance to antibiotics which interfere in cell wall synthesis, such as glycopeptides ${ }^{1}$ and lipopeptides, ${ }^{2,3}$ but also in strains exposed to protein synthesis inhibitor antibiotics, such as aminoglycosides, ${ }^{4,5}$ macrolides, ${ }^{6}$ and tetracyclines. ${ }^{7-9}$
Correspondence: Francisco Javier Candel Department of Clinical Microbiology, Hospital Clínico San Carlos, Health Research Institute, Universidad Complutense, Avda, Profesor Martín Lagos s/n, 28040 Madrid, Spain

Tel +34 9l 3303486

Fax +34913303478

Email fj.candel@gmail.com 
A methicillin- and linezolid-resistant S. aureus (MLRSA) outbreak that occurred in our hospital (Hospital Clínico San Carlos, Madrid, Spain) in 2010 allowed us to characterize some genetic resistance mechanisms that led to mutations in the ribosomal protein L3, which interferes with linezolid action. ${ }^{10}$ Whether these altered genetic patterns could also result in morphological changes is an interesting field of study that will complement all other findings.

The aim of this study was to examine cell wall and septum thickening of methicillin-sensitive $S$. aureus (MSSA), methicillin-resistant S. aureus (MRSA), and MLRSA isolates by transmission electron microscopy (TEM).

\section{Materials and methods}

Glass beads of eight different $S$. aureus frozen isolates (two MSSA, three MRSA, three MLRSA) collected from patients' samples were plated on Columbia sheep blood agar (bioMeriéux, Marcy l'Étoile, France) and incubated overnight at $37^{\circ} \mathrm{C}$. Identifications were confirmed by mass spectrometry (MALDI-TOF, Bruker, Germany). Susceptibility against oxacillin, vancomycin, daptomycin, linezolid, tetracycline, erythromycin, amikacin, and gentamycin was determined using Etest and microdilution (Vitek ${ }^{\circledR} 2$ compact; bioMérieux). Resistance and susceptibility were confirmed by Clinical Laboratory Standards Institute interpretation of microdilution and Etest minimal inhibitory concentration (MIC) results. ${ }^{11}$

TEM was used to characterize cell wall and septum thickening in all isolates. S. aureus isolates were harvested and washed with Millonig's buffer solution. Isolates were later pelleted and treated with Karnovsky's fixer solution followed by $1 \%$ osmium tetroxide solution. Pellets were dehydrated with graded concentrations of acetone and finally embedded in resin. Ultrathin samples were cut on an ultramicrotome, stained with uranyl acetate, and examined under a JEM 1010 (JEOL, Tokyo, Japan) TEM.

Cell wall and septum images were captured at 1,50,000× magnification. An average of 40 cells per bacterial strain were examined and wall and septum thickness were measured at nearly equatorial cut surfaces by using ImageJ 2.0 (NIH, Bethesda, MD, USA). Results are expressed as mean \pm SD and statistical analysis consisting on a Dunn-Bonferroni's nonparametric comparison for post hoc testing after a statistically significant Kruskal-Wallis test was performed using SPSS 20 (IBM, Chicago, IL, USA). The level of significance was defined as $P<0.05$.

\section{Results}

All MSSA isolates were sensitive to all antibiotics tested. One out of three MRSA isolates was resistant to at least one aminoglycoside and the rest were resistant to erythromycin. All MLRSA isolates were resistant to erythromycin and to at least one aminoglycoside. None of the MRSA or the MLRSA strains were resistant to tetracycline.

The mean cell wall and septum thickness measurements obtained through TEM were, respectively, 17.20 \pm 2.58 and $29.17 \pm \mathrm{Y} \mathrm{nm}$ for the MSSA isolates, 24.42 \pm 4.64 and $47.21 \pm 10.30 \mathrm{~nm}$ for the MRSA isolates, and 23.54 \pm 3.83 and $51.82 \pm 9.02 \mathrm{~nm}$ for the MLRSA isolates. Differences in cell wall and septum thickness were statistically significant ( $P<0.001$ and $P<0.001$, respectively) between the three groups. By means of a pairwise comparison, significant differences were detected in wall thickness between the MSSA and MRSA strains $(P<0.001)$ and between the MSSA and MLRSA strains $(P<0.001)$ but not between the MRSA and MLRSA strains $(P=0.386)$. In the case of the septum thickness, significant differences were observed between the MSSA and MRSA strains $(P<0.001)$ and between the MSSA and MLRSA strains $(P<0.001)$ but not between the MRSA and MLRSA strains $(P=0.117)$. Results are summarized in Figure 1.

\section{Discussion}

There are several mechanisms explaining cell wall thickening. Decreased activity of penicillin binding proteins (PBP) affects crosslinking, producing an excess of terminal D-alanyl -D-alanine amino acid residues. In other cases, enhanced expression of genes associated with cell wall synthesis led to an increased production of wall teichoic acid. As a result, antibiotics delay their access to active sites in cell wall synthesis (particularly in the septum). These events are described in $S$. aureus isolates with decreased susceptibility to cell wall synthesis inhibitors, such as vancomycin ${ }^{1,12}$ and daptomycin. ${ }^{2}$ Heterogeneous vancomycin-intermediate Staphylococcus aureus (hVISA) strains with mutations in either the graRS, vraSR, or walKR operon (or all) that might lead to their respective regulons remaining in an activated "locked-on" or otherwise modified state. The consequence of this modification includes cell wall thickening, decreased autolysis, reduced protein A production, increased capsule expression, increased D-alanylation of teichoic acids, and reduced agr gene activity. ${ }^{13,14}$ In this study, we observed that MRSA had thicker walls than MSSA. High vancomycin and daptomycin MIC found in MRSA could be an explanation for this thickening.

Defects in protein functions can also take a part in this mechanism of resistance. A decreased activity of diverse autolysins of S. aureus could lead to a decreased peptidogly- 

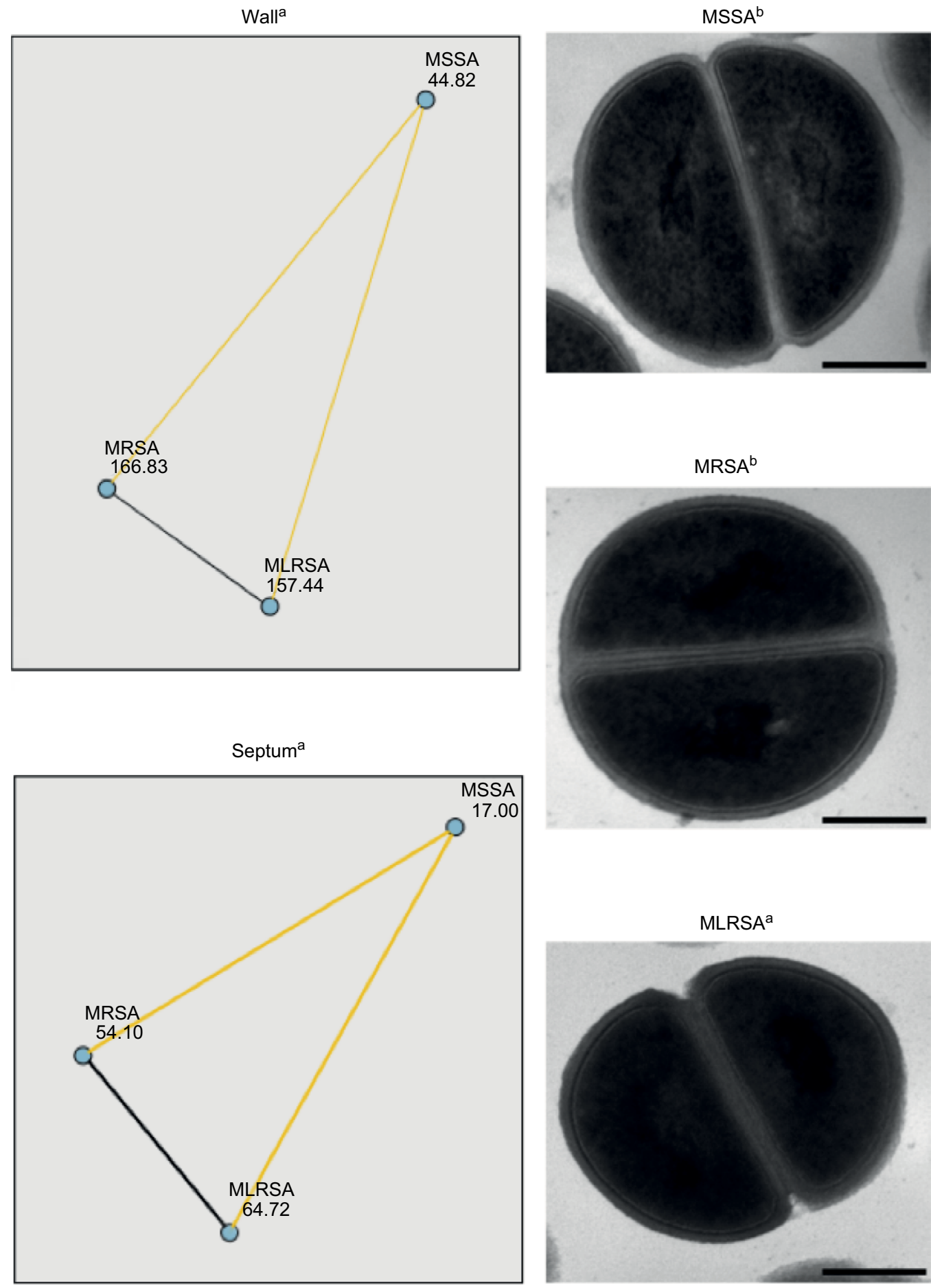

Figure I Graphical representation of Dunn-Bonferroni's nonparametric pairwise comparison for post hoc testing after a significant Kruskall-Wallis test of MSSA, MRSA, and MLRSA wall and septum measurements, and TEM images of a representative example of each group.

Notes: a Numerical values represent the mean rank of each group. Yellow lines represent statistically significant $(P<0.05)$ comparisons between the groups they connect. Black lines represent nonsignificant $(P \geq 0.05)$ comparisons between the groups they connect. bMagnification: I,50,000×. Scale bar: 200 nm.

Abbreviations: MLRSA, methicillin- and linezolid-resistant Staphylococcus aureus; MRSA, methicillin-resistant S. aureus; MSSA, methicillin-sensitive S. aureus; TEM, transmission electron microscopy.

can lysis. This is observed in the presence of tetracycline, a protein synthesis inhibitor. ${ }^{89}$ When necessary proteins for cell wall synthesis, such as kinases and phosphatases, are absent, defects in peptidoglycan linking and thickened cell walls are observed. ${ }^{15}$ This antibiotic group may affect synthesis and metabolism of these kinds of proteins, collaborating in cell wall thickening. But at present, whether this effect is a result of protein synthesis inhibitors or whether previous changes, 
such as mutations in ribosome or enzymatic modifications, could cause resistance to these antibiotics and cell wall changes at the same time is not clear. ${ }^{4,6}$ We thought that linezolid, as a protein synthesis inhibitor, could collaborate in this process.

Further studies focusing on ribosome, protein function, and cell wall structure are necessary to better establish the relationship between protein synthesis inhibitors and cell wall metabolism.

\section{Acknowledgment}

This study has been funded by grant PI13/01471 as part of the National R and D Plan, AES 2013 and co-funded by the ISCIII and the European Regional Development Fund (ERDF).

\section{Disclosure}

The authors report no conflicts of interest in this work.

\section{References}

1. Cázares-Domínguez V, Cruz-Córdova A, Ochoa SA et al. Vancomycin tolerant, methicillin-resistant Staphylococcus aureus reveals the effects of vancomycin on cell wall thickening. PLoS One. 2015;10(3): e0118791.

2. Bertsche U, Yang SJ, Kuehner D et al. Increased cell wall teichoic acid production and D-alanylation are common phenotypes among daptomycin-resistant methicillin-resistant Staphylococcus aureus (MRSA) clinical isolates. PLoS One. 2013;8(6):e67398.

3. Bayer AS, Schneider T, Sahl HG. Mechanisms of daptomycin resistance in Staphylococcus aureus: role of the cell membrane and cell wall. Ann NY Acad Sci. 2013;1277:139-158
4. Fukutsuji K, Yamada S, Harada T. Ultrastructural cell wall characteristics of clinical gentamycin-resistant Staphylococcus aureus isolates. Med Mol Morphol. 2013;46(2):70-76.

5. Yuan W, Hu Q, Cheng $\mathrm{H}$ et al. Cell wall thickening is associated with adaptive resistance to amikacin in methicillin-resistant Staphylococcus aureus clinical isolates. J Antimicrob Chemother. 2013;68(5): 1089-1096.

6. Hyo Y, Yamada S, Fukutsuji K, Harada T. Thickening of the cell wall in macrolide-resistant Staphylococcus aureus. Med Mol Morphol. 2013;46(4):217-224.

7. Hash JH, Wishnick M, Miller PA. On the mode of action of tetracycline antibiotics in Staphylococcus aureus. J Biol Chem. 1964;239: 2070-2078.

8. Ledala N, Wilkinson BJ, Jayaswal RK. Effects of oxacillin and tetracycline on autolysis, autolysin processing and atl transcription in Staphylococcus aureus. Int J Antimicrob Agents. 2006;27(6):518-524.

9. Yamada S, Sugai M, Komatsuzawa H, Matsumoto A. Suppressed localization of a major autolysin on Staphylococcus aureus treated with tetracycline. J Electron Microsc (Tokyo). 2001;50(4):359-364.

10. Morales G, Picazo JJ, Baos E et al. Resistance to linezolid is mediated by the cfr gene in the first report of an outbreak of linezolid-resistant Staphylococcus aureus. Clin Infect Dis. 2010;50(6):821-825.

11. CLSI. Performance Standards for Antimicrobial Susceptibility Testing. 27th ed. CLSI supplement M100. Wayne, PA: Clinical and Laboratory Standards Institute; 2017.

12. Gardete S, Tomasz A. Mechanisms of vancomycin resistance in Staphylococcus aureus. J Clin Invest. 2014;124(7):2836-1240.

13. Howden BP, Davies JK, Johnson PD, Stinear TP, Grayson ML. Reduced vancomycin susceptibility in Staphylococcus aureus, including vancomycin-intermediate and heterogeneous vancomycin-intermediate strains: resistance mechanisms, laboratory detection, and clinical implications. Clin Microbiol Rev. 2010;23(1):99-139.

14. Howden BP, McEvoy CR, Allen DL, et al. Evolution of multidrug resistance during Staphylococcus aureus infection involves mutation of the essential two component regulator WalKR. PLoS Pathog. 2011;7(11):e1002359.

15. Beltramini AM, Mukhopadhyay CD, Pancholi V. Modulation of cell wall structure and antimicrobial susceptibility by a Staphylococcus aureus eukaryote-like serine/threonine kinase and phosphatase. Infect Immun. 2009;77(4):1406-1416.
Infection and Drug Resistance

Publish your work in this journal

Infection and Drug Resistance is an international, peer-reviewed openaccess journal that focuses on the optimal treatment of infection (bacterial, fungal and viral) and the development and institution of preventive strategies to minimize the development and spread of resistance. The journal is specifically concerned with the epidemiology of antibiotic

\section{Dovepress}

resistance and the mechanisms of resistance development and diffusion in both hospitals and the community. The manuscript management system is completely online and includes a very quick and fair peerreview system, which is all easy to use. Visit http://www.dovepress.com/ testimonials.php to read real quotes from published authors. 